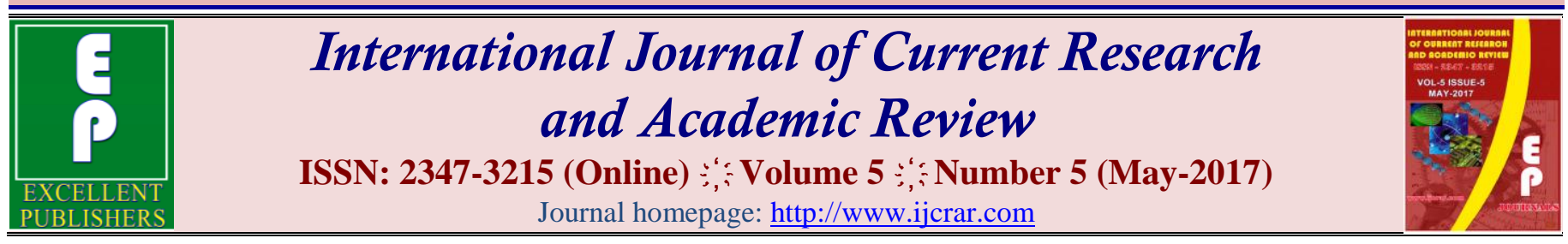

doi: $\underline{\text { https://doi.org/10.20546/ijcrar.2017.505.011 }}$

\title{
Prevalence and Risk Factors of Low Back Pain among Auto-Rickshaw Drivers in Urban Kolkata, India
}

\author{
Shabnam Agarwal*, Anwesh Pradhan, Gargi Ray Chaudhuri and Soumitra Das \\ Nopany Institute of Healthcare Studies, Chief Physiotherapist, Belle Vue Clinic, Kolkata, India \\ *Corresponding author email
}

\begin{abstract}
Auto rickshaw drivers are prone to Low Back Pain (LBP) due to occupational exposure. But LBP among auto-rickshaw drivers have hardly been studied. The drivers work long durations in strenuous postures and seat themselves often with onetwo passengers in front. The present study was to determine the prevalence and risk factors of LBP in auto-rickshaw drivers of Kolkata.500 auto-rickshaw drivers were randomly surveyed in auto-stands of various parts of Kolkata. A Nordic Pain questionnaire was used to determine prevalence, the 101 Numerical Pain Rating Scale measured current pain intensity and the Oswestry Disability Index evaluated disability due to LBP. Risk factors included age, height, BMI, socio-demographic and occupational details, shoulder to handle distance, knee to break distance, pain intensity, vibration and posture during driving. The Ovako Working postural Analysis System (OWAS) was used to analyze the posture during driving and a Vibrometer was used to analyze the vibration. Logistic regression analysis was performed to ascertain the association of risk factors with LBP. A Pearson's chi square test was performed to determine the association between two categorical variables. The 12 months prevalence of LBP was $79.8 \%$ and 7 days point prevalence was $36 \%$. Forward bent and twisted sitting posture (OWAS action level 2) was significantly associated with LBP.
\end{abstract}

\section{Article Info}

Accepted: 05 May 2017

Available Online: 20 May 2017

\section{Keywords}

Auto-rickshaw,

Drivers, Kolkata,

Low back pain,

Occupational low back pain,

OWAS, Prevalence,

Risk factors,

Posture while driving.

\section{Introduction}

The worldwide annual incidence of Low backs pain (LBP) ranges from $1.5 \%$ to $36 \%$, and the one year prevalence ranges from $22 \%$ to $65 \%$ (Hoy et al., 2010). The recurrent episodes of LBP range from $24 \%$ to $33 \%$ (Stanton et al., 2008).

LBP amongst drivers is common due to several reasons. Driving is a task which involves prolonged sitting, awkward posture, static position and vibration; any of which could directly lead to musculoskeletal stress. The results of the prevalence studies of LBP in heavy vehicle drivers vary between the type of vehicles and from the countries they are reported from (Olanrewaju et al., 2007). The point prevalence and 12 months prevalence in Danish fork-lift truck drivers is $21 \%$ and $65 \%$ respectively (Sami et al., 2012) while in a Finnish study of earth mover operators, it is higher at $51 \%$ and $82 \%$ respectively (Olanrewaju et al., 2007). Among the bus drivers, prevalence of musculoskeletal disorders and low back troubles varies between $40 \%$ to $82 \%$. The 12 months prevalence of LBP in taxi drivers is lower compared to heavier vehicle drivers and varies between $59 \%$ in Malaysia (Sami et al., 2012) to $46 \%$ in Southampton, UK (Gallais et al., 2008). One would expect the prevalence of LBP to reduce further in lighter vehicle, as in an autorickshaw. To the contrary, it is 
observed that the 12 months prevalence of LBP in autorickshaw drivers in Guntur, India, is as high as $63.66 \%$ (Shaik et al., 2014).

The etiological factors of LBP include poor posture, muscle weakness and imbalance, (Shaik et al., 2014) degenerative changes in the disc and vertebral bodies, trauma, anxiety and depression (Stanton et al., 2008). Risk factors are characteristics, specific to people that have been correlated to incidents of LBP. The risk factors associated with LBP include age, gender, anthropometrics, overall health (Khan et al., 2014), and socioeconomic status (Stanton et al., 2008), education levels (Samsul et al., 2007, Dionne et al., 2006), smoking habits (Racheal, 2014), whole body vibration (Gregory, 2008; Charoenchai et al., 2006) and awkward occupational postures, work experience (number of years), working hours, seat vibrations, lower cabin space and shoulder to handle distance for right and left upper limbs (Shaik et al., 2014).

Scarce number of studies have been conducted or reported on auto-rickshaw drivers. In India many cities have this mode of transport. In Kolkata, auto-rickshaws were introduced in 1983-84 and the current fleet of registered vehicles is 10,000 (The Hindu Magazine, 2014).

Out of the two previous studies on auto-rickshaw drivers, one was conducted in Guntur, Andhra Pradesh, India (Shaik et al., 2014)) and the other in Galle, Sri Lanka (Kirkorowicz et al., 2013). Both studies conclude the driver's seat vibration as a risk factor for LBP. Further, the study by (Kirkorowicz et al., 2013), attributed musculoskeletal pain in lower back, shoulders and knees to staying in the same posture for many hours. Long duration cramped sitting work postures in autorickshaw drivers may cause a significant static loading on the structures of the spine (Odebiyi et al., 2007). In addition twisting and bending of vertical column (Rajnarayan et al., 2003), spinal instability (Panjabi, 2003), intra-discal pressure, hydrostatic properties of the disc (Nachemson, 1975), muscle fatigue due to lack of oxygenation are also included as an important causes of LBP.

Given the limited amount of previous research, inadequate assessment of risk factors, small sample size, it is evident that a survey is required which explores all relevant risk factors in an adequate sample of autorickshaw drivers. Further, considering the growing popularity of this transportation system, the increasing population and traffic congestion in India, the auto- rickshaw drivers' health issues require attention. The results could prove to be useful in government analysis and policy development concerning auto-rickshaw drivers. Hence, the objectives of this study were (1) To determine the prevalence of LBP in auto-rickshaw drivers in urban Kolkata (2) To explain the risk factors, which may contribute to the development of LBP in auto-rickshaw drivers in urban Kolkata.

\section{Materials and Methods}

The study design was a descriptive type survey using cluster sampling. The survey method was mainly in the form of questionnaires which were interviewed in person, individually to each participant. A total of 575 auto-rickshaw drivers were contacted. 65 declined participation while 10 terminated participation. Eventually 500 auto-rickshaw drivers from different routes of urban Kolkata were surveyed for this study (Figure 1). Approval for the research was obtained from the Institutional Human Research Ethics Committee (meeting on 28 03.2015). Informed consent forms (Bengali, Hindi or English) were signed by all participants prior to the survey.

Parameters studied for the participants were intraindividual (socio-demographics), occupational (work experience as in number of years, working hours per day, working hours per week, shoulder to handle distance, knee to brake distance), posture, pain site, pain intensity, vibration and disability. The plan of the survey is illustrated in figure 2.

\section{Outcome measures}

Every participant was assessed using the following outcome measures:

Socio-demographics for intra-individual and occupational data included questions about age (years), gender (male), BMI, currently smoking (Yes/ No), education levels, marital status, (work experience in number of years, working hours per day, working hours per week, shoulder to handle distance $(\mathrm{cm})$, knee to brake distance $(\mathrm{cm})$. Data type was nominal or interval.

Nordic Musculoskeletal Questionnaire (NMQ): NMQ (Kuorinka et al., 1987) was used to ascertain presence, extent and prevalence of LBP. The subcomponents of the NMQ can justify the low back pain properly.For participants who did not understand English or could not read the questionnaire was used in an interview format. 
A Bengali version was used in such cases. The reliability and validity of the Bengali version was not tested. However, the translation was done by a language expert and then tested on three pairs of medical professional by the back and forth method.

Ovako Working postural Analysis System (OWAS): It is a method of coding the posture of a worker that allows the harmfulness of the posture to be categorized into four action categories of increasing urgency and has been found reliable (Brujin et al., 1998). In this study two action levels were considered.

OWAS action level 1 - sitting posture of auto-rickshaw driver. Level 1definition implied the driver's back posture was 'straight'

OWAS action level 2 - sitting posture of auto-rickshaw driver. Level 2 definition implied theback posture as 'bent and twisted'.

101 Numerical Pain Rating Scale (101 NPRS): The 101 NPRS was used for the drivers to record their perceived level of pain intensity on a numerical scale from 0-100, $(0=$ no pain and 100 representing maximum pain). The scale is practical, easy to administer and sensitive (Williamson et al., 2005; Jenson et al., 2011).

Vibrometer (android application): An android Vibrometer (1.4.6) was used for measuring the vibration of vehicles. Data type was interval. A Vibrometer application was used when the vehicle was in motion for a minimum period of 5 minutes and a maximum period of 20 minutes. The device was kept at the junction of the driver's seat and backrest to measure the vibration when the auto-rickshaw was full of passengers. The maximum vibration (in hertz) was noted.

Oswestry Disability Index (ODI): The ODI is an evaluative, self-administered questionnaire for scoring the disability of patients with LBP and indicates the extent to which a person's functional level is restricted by back pain. The reliability and validity of the ODI has been established (Fairbank et al., 2000). Ten stem questions which form the ODI are pain intensity, personal care (washing, dressing), lifting, walking, sitting, standing, sleeping, sex life, social life and travelling. ODI questions number 8 and 9 were omitted as they related to 'Sex life and Social life'. Hence the total score was out of 40 instead of 50 and the percentage of disability was calculated accordingly. The ODI was translated into Bengali using the same method as the NMQ. Administration method was the same as well.

\section{Statistical analysis}

Logistic regression analysis was performed to ascertain the association of all risk factors with LBP (dependent variable).The Pearson's Chi square test was conducted to test the association between categorical variables and LBP prevalence. The significance level for all analysis was determined at $\mathrm{p}<0.05$.

\section{Results and Discussion}

The first question of the NMQ asks if the participant has ever had LBP. $411(82 \%)$ out of 500 auto rickshaw drivers said "yes". $399(79.8 \%)$ had pain in the last 12 month period. The data representing LBP troubles of last 12 months was considered for statistical analysis in the study. The 7 days point prevalence is $36 \%$. Results are approximately similar to that of Shaik et al., (2014), where the 12 months prevalence of LBP is $63.66 \%$. One of the reasons for auto-rickshaw drivers to exhibit a high prevalence could be that they tend to sit in awkward postures resulting in musculoskeletal disorders while driving (Nahar et al., 2012; Bovenzi et al., 2006; Borle et $a l ., 2012$ ). A further add on is that the drivers often share the driver's seat in front with passengers in order to carry more passengers and earn more. Sustained continuous sitting in awkward postures may be contributory (Ganmgopadhyay and Dev., 2012).

In this study the current pain intensity was measured using the 101 NPRS. The pain intensity reported by the auto-rickshaw drivers is $41( \pm 18)$, range 0 - 80 .

Disability is measured using the ODI. ODI scores revealed that out of 399 auto-rickshaw drivers, 104 have no current LBP whilst 295 drivers have varying intensities of current LBP (pain intensity $1=165$ persons; $2=94$ persons; $3=32$ persons; $4=4$ persons). Out of 295 drivers, $284(71.17 \%)$ are in the minimal disability level category $(0-20 \%), 90(23.55 \%)$ are in the moderate disability level category (21-40\%) and $25(6 \%)$ were in severe disability level. Out of all the 8 stem questions of the ODI which are asked, namely, pain intensity, personal care (washing, dressing), lifting, walking, sitting, standing, sleeping, and travelling, it is observed that the sitting activity and pain has the least number of drivers at ' 0 ' level. $74 \%$ drivers have some level of pain; out of which sitting is a problem for $52 \%$ drivers, walking is a problem for $42 \%$ drivers, lifting is a problem with $31 \%$ and standing for $30 \%$. The high 
percentage of disability in sitting may be attributed to long hours of driving which the drivers do. In the study among bus drivers of urban Kolkata (26 Gangopadhyay and Dev, 2012) cramped sitting posture is the main cause of LBP.

Several risk factors have been associated with LBP in drivers and are explored and evaluated in this study as well.

The mean age of the respondents is 40 years, range between 27 to 56 years. This age group is similar to that noted in previous studies on car drivers (mean age $=43$ years) and auto-rickshaw drivers (mean age 46 years) (Kirkorowicz et al., 2013). Older age has been associated as a risk factor for LBP in car drivers (Nahar et al., 2012).Whilst previous studies on auto-rickshaw drivers explored age as a risk factor, they did not find any significant association (Shaikh et al., 2014). The results of this study did not find any significant association between age and LBP either, although drivers who have current pain and disability are more in the age group 3456years.

The mean height of the surveyed driver is 164 centimeters, weight 68 kilograms, and BMI is 25.Anthropometrics have not been studied in previous surveys on auto-rickshaw drivers (Shaik et al., 2014; Kirkorowicz et al., 2013). A previous study on bus drivers of Kolkata, (Gangopadhyay and Dev, 2012) revealed that the average height of bus drivers was 162 $\mathrm{cm}$, weight $53 \mathrm{~kg}$ and BMI 20. The results of this survey measured the average height of auto-rickshaw drivers as similar; $164 \mathrm{~cm}$. However, they weighed much more than their bus counterparts; mean weight is $68 \mathrm{~kg}$ and the $\mathrm{BMI}$ is consequently higher as well (mean $=25$, range $=$ 20 - 35). Since, both kinds of drivers come from the same population, it is not clear why the auto-rickshaw drivers weighed more with a BMI category as 'overweight'.

$37.3 \%$ responders received education till the secondary level (i.e. till class $\mathrm{x}$ ); however nearly an equal number are uneducated $(31.4 \%)$. Only a small number (1.2\%) reached graduation qualification. The one previous study (Kirkorowicz et al., 2013) conducted in Galle which recorded the education levels of their participants has a similar distribution. However, comparisons are difficult as their sample size is extremely small. Looking at a study on bus drivers of Malaysia, a similar education qualification population distribution is seen for primary (31.2\%), secondary (36.4\%) and graduate levels (2.5\%) (Shamsul et al., 2007). A review study by Dionne et al.,
(2001) associated low education qualifications with adverse consequences of LBP. Various factors are considered to contribute to this. However, similar to the results of this study, a previous study (Shamsul et al., 2007) on commercial drivers demonstrated no significant association between LBP and educational levels.

The percentage of drivers who are married are $62 \%$ which is less than reported in previous survey studies where the average percentage of married drivers are $72 \%$ (Bovenzi et al., 2006) and 90\% (Kirkorowicz et al., 2013). A study on truck drivers in Nagpur (Borle et al., 2012) shows no significant association between marital status and LBP, which was observed in this study as well.

Smoking is a common habit among drivers. In this study $81 \%$ drivers are smokers similar to studies in Sri Lanka which reports $98 \%$ auto-rickshaw drivers as smokers (Kirkorowicz et al., 2013) and in Bangladesh where 80\% professional car drivers were smokers. Although it is an established fact that smoking is a risk factor for musculoskeletal problems since it has a negative impact on bone mineral density and causes lowering of vitamin D levels, changing hormones levels, slowing healing process and increases fracture chances by $40 \%$ (Racheal, 2014), the results of this study as well as that of Nahar et al., (2012) show no significant association with LBP in drivers.

Hours of driving and its correlation with LBP has been primarily explored in bus drivers (Gangopadhyay et al., 2012) and found significant (Odebiyi et al., 2007), this study (Table 1) as well as a previous study on autorickshaw drivers found no association(Shaikh et al., 2014).

The auto-rickshaw drivers in this study have an average driving experience of $14( \pm 56)$ years, similar to a previous study reported as 16 years (Nasrin et al., 2012) but more than that reported by Kirkorowicz et al., (2013) 9 years. This study does not find any significant association between number of years of experience and LBP. The results are similar to the study from Guntur (Shaik et al., 2014).

Heavy vehicle drivers are more prone to whole body vibrations which cause LBP. Vibration measured from driver's seat shows increase risk of low back injury. Studies say vibration of vehicle in a hertz of $4 \mathrm{~Hz}$ and above stimulated back muscle fatigue and cause back pain. A previous study found vibration as a risk factor 
for LBP in auto-rickshaw drivers. However, the results of such an association are not significant in this study. A possible reason may be the android Vibrometer which is an amateur device and whose reliability and validity has not been tested.

Other risk factors explored in previous surveys (Shaik et al., 2014; Kirkorowicz et al., 2013) are shoulder to handle distance and lower cabin space. In this study too, these risk factors are analyzed but no significant associations are seen similar to the results of the previous studies.

The logistic regression analysis showed that none of the independent variables were risk factors for LBP. The Pearson's Chi square test as well as the Phi test for association between two nominal variables (LBP and posture) were significant $(\mathrm{p}<0.05)$ between LBP and posture (Table 2). 81\% auto-rickshaw drivers at action level 2 reported 'yes' to LBP. The results implied that these were a significant difference in the prevalence of LBP between auto-rickshaw drivers who sat 'straight' (OWAS action category 1) compared to those who sat 'bent forward and twisted' while driving (OWAS action category 2). This indicates that drivers who are in a posture described in the scale as (the drivers have their back posture as bent and twisted, upper limbs (arms) below shoulder level, lower limbs (legs) are hanging free and use of force needed $10 \mathrm{~kg}$ or less) are at a higher risk of having LBP than drivers who are categorized as (drivers having their back posture straight, upper limbs (arms) below shoulder level, lower limbs lower limbs (legs) are hanging free and use of force needed is $10 \mathrm{~kg}$ or less). Category action 1 means 'posture is acceptable' and category action 2 means 'Some strain- action or investigation is required'. In category action 1, those having LPB are $77(57.3 \%)$ drivers and those in category action 2 are $215(81.43 \%)$ drivers.

Previous literature indicates that working posture in which the individual is mostly bending or rotating, is in flexion or lateral bending, is twisting and or is in an awkward posture, doing the same activity continuously, eventually is a cause for muscle fatigue (Charoenchai et al., 2006; Nasrin et al., 2012). Sedentary work like driving causes LBP, where driver's hips at an angle of $90^{\circ}$ or less, produces continuous pressure over lumbar discs which may lead to degeneration of the lumbar spine. A study on bus drivers of Kolkata (Gangopadhyay and Dev, 2012) evaluated torso posture in as much details as the current study and similar to the results of the study concluded that poor posture while driving is a significant risk factor in LBP.

Table.1 Occupational (Work-style) details of auto-rickshaw drivers in Kolkata $(n=399)$

\begin{tabular}{lcc}
\hline \multicolumn{1}{c}{ Work style details } & Mean $( \pm \mathrm{SD})$ & Range \\
\hline Driving experience (in years) & $14 \pm 5.6$ & $3-35$ \\
Driving hours per day & $11 \pm 1.6$ & $7-16$ \\
Driving hours per week & $69 \pm 12$ & $40-112$ \\
Shoulder to handle distance (in cm) & $50 \pm 3.5$ & $38-63$ \\
Knee to brake distance (in cm) & $46 \pm 3$ & $37-57$ \\
Vibration measurement (in Hertz) & $8.1 \pm 61$ & $6-10$ \\
\hline
\end{tabular}

Table.2 Result of the Pearson's Chi square test to test the association between posture (OWAS) and LBP

\begin{tabular}{cccccc}
\hline Risk factor & \multicolumn{2}{c}{ LBP } & Total & $\begin{array}{c}\text { Pearson's chi } \\
\text { square }\end{array}$ & Phi \\
\hline & No (\%) & Yes (\%) & & & \\
Posture & & & & 0.487 & 0.264 \\
(OWAS) & $58(42.9)$ & $77(57.03)$ & 135 & & \\
Level 1 & $49(18.56)$ & $215(81.43)$ & 264 & & \\
Level 2 & & & & \\
\hline
\end{tabular}


Figure.1 OWAS action level 1 - sitting posture of auto-rickshaw driver of urban Kolkata: OWAS action level 2 sitting posture of auto-rickshaw driver of urban Kolkata

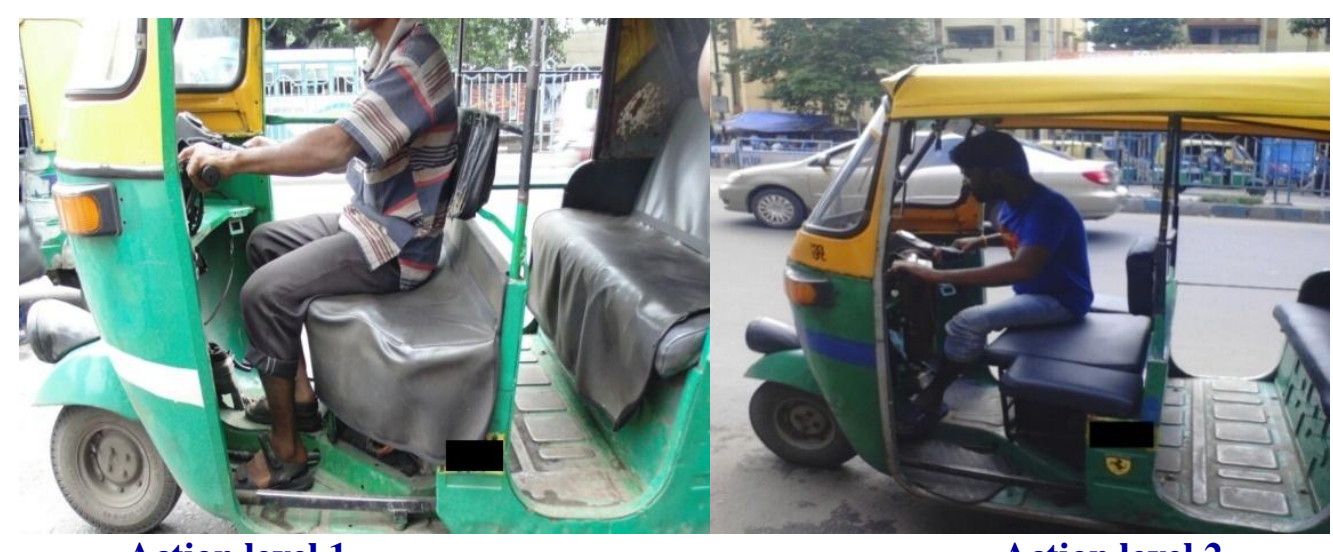

Action level 1

Action level 2

Figure.2 Research plan

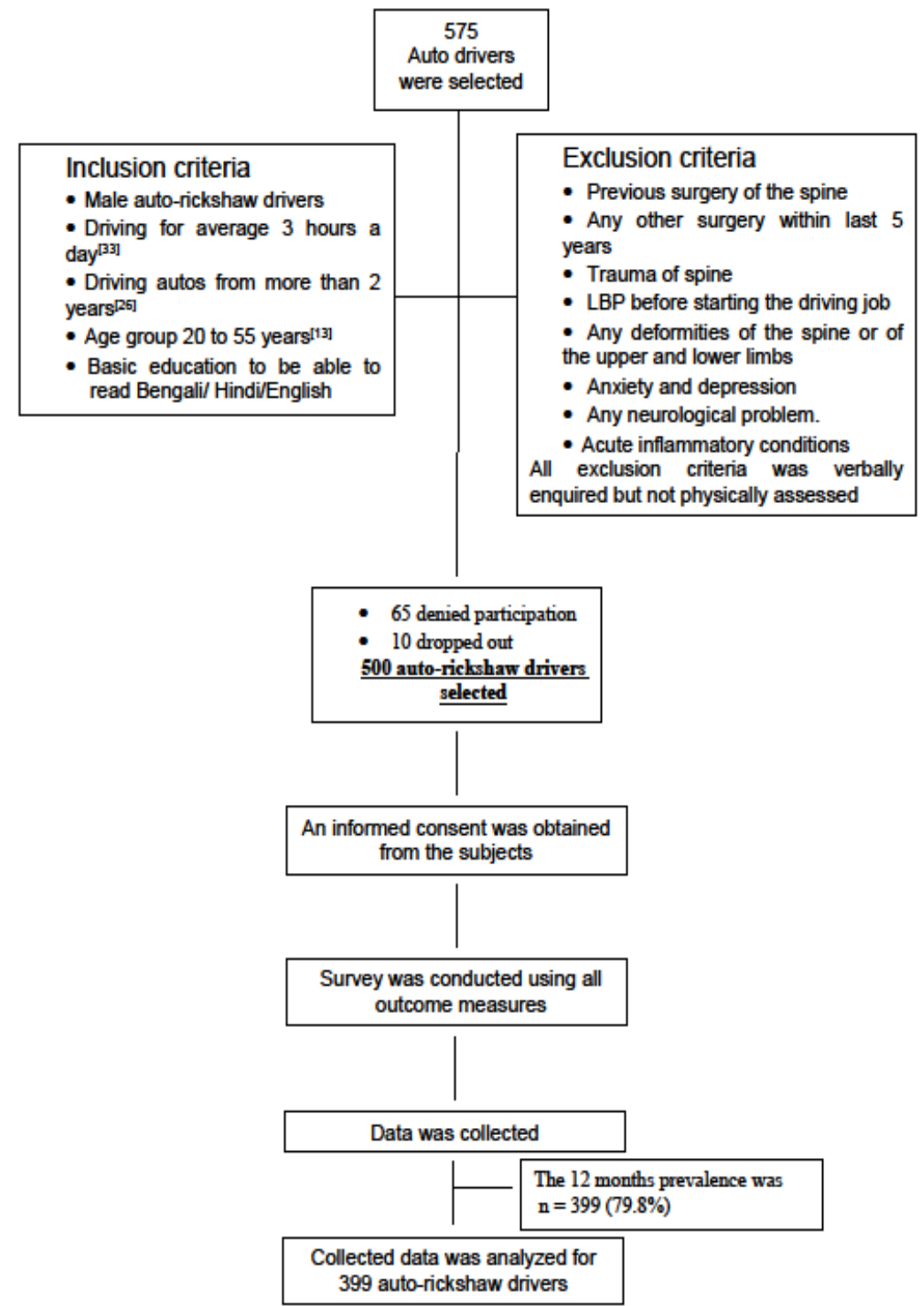




\section{Conclusion}

The prevalence of LBP in auto-rickshaw drivers of urban Kolkata is higher than the LBP prevalence in other vehicle drivers. The risk factor for LBP in autorickshaw drivers is the forward bent and twisted sitting posture which is frequently adopted by auto-rickshaw drivers while driving.

\section{Recommendations for future research}

- More auto routes may be surveyed.

- The same type of auto models should be selected.

- Measurements for the upper limbs (shoulder to handle distance) should have be taken for the both sides.

- Vibration measurement tool should be tested for accuracy and reliability.

- The survey population could have been more in number.

\section{References}

Hoy D, Brooks P, Blyth F, Buchbinder R. 2010. The epidemiology of LBP. Best Practice Res. Clin. Rheumat., 24:769-81

Stanton TR, Henchke N, Maher CG. 2008. After an episode of acute LBP, recurrence is unpredictable and not as common as previously thought. Spine, 33:2923-8.

Olanrewaju O, Steven JS, Marianne M, Malcolm P. City bus driving and LBP- A study of the exposures to posture demands, manual materials handling and whole-body vibration. Appl. Ergonomics, 38: 29-38.

Sami ARD, Ahmed MQ, Noor HI, Krishna GR. 2012. Prevalence and determinants of LBP among taxi drivers in Malaysia. A cross sectional study. $J$. Adv. Med. Res., 2:129-43.

Gallais L. 2008. LBP and risk factors for LBP in car drivers (unpublished dissertation).University of Southamptom.

Shaik R, Christie KG, Chintada GS, Sandeep R.The Prevalence of Musculoskeletal disorders and their association with Risk Factors in Auto Rickshaw Drivers-A survey in Guntur City. Int. J. Physiother., 2014; 1:2-9.

Khan. AA, Mohammad MU, Ahsanul HC, Ranjan KG. 2014. Association of LBP with common risk
Factors: A community based study. IJPMR, 25: 50-5.

Shamsul BT, Kazuhito Y, Juliana J, Nasaruddin AA, Nizam J, Rusli N, et al. 2007. The Association between Risk Factors and LBP among Commercial Vehicle Drivers in Peninsular Malaysia: A Preliminary Result. Industrial Health, 45:268-78.

Dionne CE, Dunn KM, Croft PR. 2006. Does back pain prevalence really increase with increasing age? A systemic review. Age Age., 35: 229-34.

Racheal M. The role of smoking in the progressive decline of the body's major systems. Public Health England. 2014: 597-607

Gregory PS. Effects of Seated Whole-Body Vibration on Spinal Stability Control. Virginia Polytechnic Institute and State University. 2008.

Charoenchai L, Chaikoolvatana A, Chaiyakul P. The relationship between health behaviour and pain scale in patients with LBP in Thailand, Department of Pharmacological science, UbonRatchathani University, Ubon Ratchathani, Thailand, 37: 1040-8.

Booklet informing auto rickshaw drivers of laws, rights launched in Kolkata. The Hindu magazine. 2014.

Kirkorowicz J, Sapukotana P, De Silva PV, Noda M, Oliveira de PV, Ostbye T. 2013. Work-Related Stress and Substance Use as Risk Factors for Chronic Disease Among Three-Wheel Drivers in Galle, Sri Lanka: A Qualitative Study. Int. J. Occupat. Safety Health., 3: 21-4.

Odebiyi D, Ogwezi D, Adegoke B. 2007. The Prevalence of LBP in Commercial Motor Drivers and Private Automobile Drivers. Nigerian J. Med. Rehabilitat., 12: 20.

Rajnarayan RT, Mrinalini CP, Sanjay PZ. LBP among Textile Workers. Indian Journal of Occupational and Environmental Medicine. 2003; 7:27-9.

Panjabi MM, Clinical spinal instability band low back pain.J ElectromyogrKinesiol. 2003; 13:371-9.

Nachemson Alf. 1975. Towards a better understanding of low back pain: A review of the mechanics of the lumbar disc. Rheumatol. Rehabilitat., 14:129-43.

Kuorinka I, Jonsson B, Kilbom A. 1987. Standardized Nordic questionnaires for the analysis of musculoskeletal symptoms. Appl. Ergon., 18: 233-7.

Bruijn I, Engels JA, Gulden JW. 1998. A simple method to evaluate the reliability of OWAS observations. Appl. Ergon., 29:281-3. 
Williamson A, Hoggart B. 2005. Pain: a review of three commonly used pain rating scales. J. Clin. Nurs., 14:798-804.

Jenson MH, Peter MF, Dagny FH, Augusto C, Geoffery WH, Jon HL, et al. 2011. Studies comparing Numerical Rating Scale, Visual Rating Scale, Visual Analogue Scale for assessment of pain intensity in adults: A systemic Literature Review. J. Pain System, 41:1073-93.

Fairbank, Jeremy CT, Paul B. 2000. The Oswestry Disability Index. Spine, 25:2940-53.

Nahar NB, Ahsam GU, Nazmul AK. 2012. Prevalence of LBP and associated risk factor among any professional car drivers in Dhaka city Bangladesh. SEAL Public Health, 2: 60-3.

Bovenzi M, Corrado N, Giuliano AF, Francesca R, Sandra B, Lucia B, et al. 2006. An epidemiological study of LBP in professional drivers. J. Sound Vibrat., 298: 514-39.

Borle, A., Agawane, S., Gunjal, S., Tayde, P. 2012. Study of Occupational Factors Associated with LBP in Truck Drivers of Nagpur City, India. Int. J. Med. Health Sci., 1: 53-60.

Gangopadhyay S, Dev S. 2012. Effect of LBP on social and professional life drivers of Kolkata. IOS press and the authors. 41: 2426-33.

Nasrin S, Ehsanollah H, Seyed A.S. 2012. The relationships between musculoskeletal disorders and anthropometric indices in public vehicle drivers. Int. J. Collab. Res. Internal Med. Public Health, 4:1173-84.

Borle A, Agawane S, Gunjal S, Tayde P. 2012. Study of Occupational Factors Associated with LBP in Truck Drivers of Nagpur City, India. Int. J. Med. Health Sci., 1:53-60.

\section{How to cite this article:}

ShabnamAgarwal, Anwesh Pradhan, GargiRayChaudhuri, SoumitraDas. 2017. Prevalence and Risk Factors of Low Back Pain among Auto-Rickshaw Drivers in Urban Kolkata. Int.J.Curr.Res.Aca.Rev. 5(5), 85-92. doi: https://doi.org/10.20546/ijcrar.2017.505.011 